ISSN 0258-7122

Bangladesh J. Agril. Res. 38(4): 659-672, December 2013

\title{
EFFICACY OF FUNGICIDES AND ORGANIC OILS TO CONTROL POWDERY MILDEW DISEASE OF JUJUBE (Ziziphus mauritiana Lam.)
}

\author{
M. Z. HoQUe ${ }^{1}$, A. M. AKANDA ${ }^{2}$ M. I. H. MIAN $^{2}$ AND M. K. A. BHUIYAN ${ }^{2}$
}

\begin{abstract}
Powdery mildew (Oidium erysiphoides f.sp. ziziphi) is the major disease of Indian jujube (Ziziphus mauritiana) in Bangladesh. An experiment was conducted to test the efficacy of six fungicides, two organic oils, and a detergent against the disease. Tested fungicides were Tilt (Propiconazole) @ 0.05\%, Folicur (Hexaconazole) @ 0.1\%, Bavistin DF (Carbandazim) @ 0.2\%, Dithane M-45 (Mancozeb) @ 0.3\%, Thiovit 80 WG (Sulpher) @ 0.3\%, and Cupravit (Copper) @ 0.3\%, the oils were Mustard oil @ 0.5\% and Neem oil 0.5\%, and the Detergent (Trix) @ 0.5\%. The materials were applied as foliar spray for seven times at an interval of 15 days. All of the fungicides and two oils gave significant decrease in severity of powdery mildew and increased fruit yield of Indian jujube irrespective of varieties, locations and over times. Among the treatments, Folicur, Tilt, Thiovit, and Dithane M-45 sprays were found effective than others. The severity index values on two jujube varieties were 3.89-4.50 at Mowna and 4.00-4.53 at Ishurdi in control treatment. The severity indices were reduced to 1.45-1.96 at Mowna and 1.33-2.07 at Ishurdi due to application of Tilt, Folicur, Thiovit, and Dithane M-45, which gave increase in fruit yield over control by 68.88, 63.69, 63.04, and 54.63\% in Apple Kul and 83.25, 77.87, 77.39, and $70.36 \%$ in BAU Kul, respectively. Most of the treatments were able to reduce number of spotted fruits. The best effective fungicide was found to be Tilt followed by Folicur and Thiovit in reducing disease severity of powdery mildew as well as other fruit diseases like fruit spot and fruit rot and increase fruit yield of Indian jujube. Therefore, Tilt/Folicur may be recommended for jujube growers to control the powdery mildew disease in commercial orchard.
\end{abstract}

Keywords: Fungicides, organic, powdery mildew jujube.

\section{Introduction}

Jujube (Ziziphus mauritiana Lam.) is commonly grown in semi arid and arid regions of India and in few other countries of the world including Bangladesh and China (Pareek, 2001). Powdery mildew caused by Oidium erysiphoides f.sp. ziziphi Yan and Wang (Microsphaera alphitoides f.sp. ziziphi Griffon and Maublanc) is a major disease of jujube in India.

Powdery mildew is one of the most widespread and easily recognized diseases. Succulent tissue is more susceptible to infection. The symptoms of the

\footnotetext{
${ }^{1}$ Senior Scientific Officer, Tuber Crops Research Centre, Bangladesh Agricultural Research Institute (BARI), Gazipur-1701, ${ }^{2}$ Professor, Department of Plant Pathology, Bangabandhu Sheikh Mujibur Rahman Agricultural University (BSMRAU), Salna, Gazipur-1706, Bangladesh.
} 
disease are noticed on leaf, young branches, flowers, and newly set fruits. The whitish powdery mass develops on young leaves eventually causes defoliation. Flower infection is the most dangerous stage. Flower infection causes defoliation of flower, as a result, fruits are not set. The disease also appears in the form of white powdery spots on the fruits and covers the entire fruit surface. The spots turn into light brown to dark brown. The infected area becomes slightly raised and rough. Affected fruits either drop off prematurely or become corky, cracked, deformed and ultimately remain unmarketable. The mycelia grow externally on the host with white, single, upright conidiophores. The fungus survives in the bud wood of the host or in some alternate hosts during the absence of jujube flowers and fruits. The disease is observed on both cultivated and wild species of jujube (Mehta, 1950). The disease is causing great loss in productivity and quality of fruits. Jamadar et al. (2009) found 50-60\% loss in fruit yield due to powdery mildew disease of jujube and reduced market value of the produce.

Jujube is a popular fruit in Bangladesh and powdery mildew is a common disease of the fruit. The yield loss due to powdery mildew disease of jujube in Bangladesh is not estimated earlier. But it was assumed that yield loss was high.

Available reports reveal that fungicides may be applied to control powdery mildew of jujube (Gupta et al., 1977; Singh et al., 1995; Sharma et al., 2001). Examples of effective fungicides are Dinocap (0.2\% Karathane WP or $0.1 \%$ Karathane EC), 0.2\% Sulfex (Gupta et al., 1977; Yadav and Singh 1985; Singh et al., 1995), 0.2\% Carbendazim (Yadav et al., 1980; Singh and Sidhu, 1985) or Trideomorph (Singh et al., 1995). According to Kapoor et al. (1975) spray of $0.07 \%$ Karathane EC or $0.3 \%$ Sulfex gave satisfactory control of powdery mildew disease of jujube. He also stated that other effective fungicides for the control of powdery mildew were Dinocap $(0.1 \%)$, Carbendazim $(0.1 \%)$, wettable Sulphur (0.2\%), Trideomorph (0.1\%), Sulphur dust (250 g/tree), and Thiophenate Methyl (0.2\%). Sharma et al. (2001) evaluated Bayleton and Karathane sprays to control and found effective. Starting with the initiation of the disease, 2 to 4 sprays at 15-20 days intervals should be carried out depending upon disease intensity. Gupta et al. (1978) and Singh and Sidhu (1985) suggested that spraying should be started as soon as the disease appears on fruits of peanut size and repeated at three weeks intervals. The powdery mildew disease can be effectively managed by alternate sprays of Triadimefon at $0.1 \%$ followed by wettable sulphur at $0.3 \%$ at an interval of $12-15$ days (Jamadar et al., 2009).

Spray of $0.5 \%$ solution of baking soda may control this disease satisfactorily. Application of neem oil is another good approach to manage the disease. Weekly application of lime-sulfur or spray or dust with sulfur is very useful to control the disease (http://www.weekendgardener.net/plant-diseases/powdery-mildew110711.htm). In order to reduce the amounts of synthetic fungicides, a wide range of alternative chemicals or natural products has been tested against 
powdery mildew disease. Homma et al. (1981) reported that sodium bicarbonate reduced the incidence of powdery mildew on cucumber plants. Foliar application of phosphate potassium salts was also found to reduce powdery mildew on cucurbit crops (Menzies et al., 1992; Reuveni , 1995).

In Bangladesh, recommended methods of control against powdery mildew of jujube are not available. Presently, jujube growers of the country applied different fungicides to control Jujube diseases without knowing their effectiveness. Therefore, in most of the cases, it is found that farmers could not achieve satisfactory results.

The present study was, therefore, conducted to find out the effectiveness of foliar sprays with fungicides, organic oils, and detergent to control powdery mildew of jujube under Bangladesh conditions.

\section{Materials and Method}

The fungicides tested in the experiment were Tilt 250 EC (Propiconazole) and Folicur 250 EC (Hexaconazole), Bavistin DF (Carbandazim), Dithane M-45 (Mancozeb), Thiovit 80 WG (Sulpher), and Cupravit (Copper oxychloride), the oils were mustard oil and neem oil, and the detergent was Trix. Folicur and Tilt were applied @ 0.05\%, Bavistin @ 0.10\%, Dithane M-45@ 0.20\%, Thiovit, and Cupravit @ 0.30\%, and mustard oil, neem oil, and Trix were applied @ 0.50\%. All of the materials were applied as foliar spray for seven times starting from 1 September with an interval of 15 days. Plain water spray served as untreated control.

The experiment was conducted in established jujube orchards of the farmers at Mawna, Gazipur and Ishurdi, Pabna during consecutive years of 2007-08 and 2008-09. At each location, two orchards were selected. One of them was planted with variety BAU Kul and the other with variety Apple Kul, which is widely cultivated in Bangladesh. In the first year, the age of BAU Kul trees was one year and that of Apple Kul was two years. All cultural and intercultural operations were applied by the owners of the orchards. The experiment was laid out in a randomized complete block design with three replications. Each experimental unit was represented by two trees. Treatment means were separated by Truky's Test.

Severity of powdery mildew was indexed on a 0-5 subjective scale (Bakr et al., 2010) where, $0=$ No lesion, $1=$ Few lesions on small laterals and leaves, $2=$ Lesions on a number of laterals and leaves, $3=$ Lesions on a few fruits and main laterals and leaves, $4=$ Lesions on a number of fruits and some laterals and leaves blighted and 5= Entire inflorescence blighted without fruit set. Data on total number of fruits per plant, spotted fruits per plant, single fruit weight (g) and fruit 
yield (kg/plant) were also recorded. Fruit spot caused by other than Oidium like Colletotrichum, Alternaria, Fusarium, etc. also include in data on spotted fruits per plant. Percent reduction in disease severity and increase in yield over control were computed.

\section{Results and Discussion}

\section{Severity of powdery mildew}

All the treatments significantly reduced powdery mildew severity $(P=0.05)$ as compared to control treatment. Severity index values of powdery mildew were maximum on both jujube varieties in both the seasons at both the locations under control treatment showing severity score of 3.89-4.53. The disease severity was reduced significantly over control due to foliar sprays with six fungicides, two organic oils, and a detergent irrespective of seasons, locations, and jujube varieties with the exception. The exception included that the detergent, mustard oil, Cupravit, and Bavistin did not reduce the disease severity significantly compared to control (Table 1). At Mowna as well as Ishurdi, disease severity index values were less than 1.33-2.07 on both Apple Kul and BAU Kul during both growing seasons under the treatments with Thiovit, Tilt, Folicur, and Dithan M-45. However, their effectiveness to reduce powdery mildew severity was statistically similar. Cupravit, Mustard oil, Neem oil and Trix were found less effective (Table 1).

\section{Diseased or spotted fruits per plant}

Like disease severity, number of spotted fruits/plant was the highest under control, which was statistically similar to effectiveness of two organic oils and the detergent. Thiovit, Tilt, and Folicur significantly reduced incidence of spotted fruits irrespective of jujube varieties, locations, and seasons compared to control. Dithane M-45 also caused significant reduction in incidence of spotted fruit over control. However, Bavistin and Cupravit gave inconsistent results (Table 2).

Percent diseased fruits of jujube significantly lower in all treatments as compared of control. The effectiveness of Tilt, Folicur, and Thiovit was the best as compared to other fungicides, organic oils, and detergent. The effectiveness of Dithane M-45 and Bavistin was less than Tilt/Thiovit. Neem oil could reduce percent diseased fruits considerably but not at par with other fungicides except Cupravit. 
Table 1. Effectiveness of different treatments with fungicides, organic oils and a detergent to control powdery mildew of Jujube at two locations during 2007-08 and 2008-09.

\begin{tabular}{|c|c|c|c|c|c|c|c|c|}
\hline \multirow{4}{*}{ Treatment } & \multicolumn{8}{|c|}{ Disease severity (0-5 Scale) } \\
\hline & \multicolumn{4}{|c|}{ Mawna, Gazipur } & \multicolumn{4}{|c|}{ Ishurdi, Pabna } \\
\hline & \multicolumn{2}{|c|}{ 2007-08 } & \multicolumn{2}{|c|}{ 2008-09 } & \multicolumn{2}{|c|}{ 2007-08 } & \multicolumn{2}{|c|}{ 2008-09 } \\
\hline & Apple Kul & BAU Kul & Apple Kul & BAU Kul & Apple Kul & BAU Kul & Apple Kul & BAU Kul \\
\hline Thiovit (0.3\%) & $1.56 \mathrm{~d}$ & $1.80 \mathrm{~d}$ & $1.78 \mathrm{~cd}$ & $1.89 \mathrm{~d}$ & $1.90 \mathrm{~d}$ & $1.33 \mathrm{~d}$ & 1.93 def & $1.57 \mathrm{~d}$ \\
\hline Tilt (0.05\%) & $1.45 \mathrm{~d}$ & $1.57 \mathrm{~d}$ & $1.56 \mathrm{~d}$ & $1.73 \mathrm{~d}$ & $1.67 \mathrm{~d}$ & $1.33 \mathrm{~d}$ & $1.69 \mathrm{f}$ & $1.50 \mathrm{~d}$ \\
\hline Folicur (0.05\%) & $1.55 \mathrm{~d}$ & $1.67 \mathrm{~d}$ & $1.73 \mathrm{~cd}$ & $1.87 \mathrm{~d}$ & $1.80 \mathrm{~d}$ & $1.67 \mathrm{~cd}$ & 1.83 ef & $1.60 \mathrm{~d}$ \\
\hline Bavistin (0.1\%) & 2.11cd & $2.10 \mathrm{~cd}$ & $2.13 \mathrm{bcd}$ & $2.17 \mathrm{~cd}$ & $2.10 \mathrm{~cd}$ & $2.67 \mathrm{abcd}$ & $2.00 \mathrm{def}$ & 2.27 bcd \\
\hline Cupravit (0.3\%) & $2.33 \mathrm{bc}$ & $2.10 \mathrm{~cd}$ & $2.28 \mathrm{bcd}$ & $2.23 \mathrm{~cd}$ & $2.43 \mathrm{bcd}$ & $2.67 \mathrm{abcd}$ & $2.54 \mathrm{bcd}$ & $2.50 \mathrm{bcd}$ \\
\hline $\begin{array}{l}\text { Dithane M-45 } \\
(0.2 \%)\end{array}$ & $1.78 \mathrm{~cd}$ & $1.80 \mathrm{~d}$ & $1.90 \mathrm{~cd}$ & $1.96 \mathrm{~d}$ & $2.00 \mathrm{~d}$ & $2.00 \mathrm{bcd}$ & 2.07 def & $1.90 \mathrm{~cd}$ \\
\hline Mustard oil (0.5\%) & $2.44 \mathrm{bc}$ & $2.67 \mathrm{bc}$ & $2.61 \mathrm{bc}$ & $2.78 \mathrm{bc}$ & $2.80 \mathrm{bc}$ & $3.00 \mathrm{abc}$ & 2.78 bc & 3.07 bc \\
\hline Neem oil (0.5\%) & $2.00 \mathrm{~cd}$ & $2.20 \mathrm{~cd}$ & $2.23 \mathrm{bcd}$ & $2.30 \mathrm{~cd}$ & $2.17 \mathrm{bcd}$ & $2.33 \mathrm{bcd}$ & 2.37 cde & $2.43 \mathrm{bcd}$ \\
\hline Trix (0.5\%) & $2.89 \mathrm{~b}$ & $3.00 \mathrm{~b}$ & $3.10 \mathrm{~b}$ & $3.27 \mathrm{~b}$ & $2.90 \mathrm{~b}$ & $3.33 \mathrm{ab}$ & $3.00 \mathrm{~b}$ & $3.50 \mathrm{~b}$ \\
\hline $\begin{array}{l}\text { Control (Plain } \\
\text { water) }\end{array}$ & 3.89 a & $4.33 \mathrm{a}$ & $4.44 \mathrm{a}$ & $4.50 \mathrm{a}$ & $4.10 \mathrm{~A}$ & $4.00 \mathrm{~A}$ & $4.39 \mathrm{a}$ & 4.53 a \\
\hline CV (\%) & 11.27 & 11.67 & 14.00 & 10.03 & 11.11 & 19.85 & 8.54 & 16.90 \\
\hline
\end{tabular}

Figures within the same column with a common letter do not differ significantly $(\mathrm{P}=0.05)$. 
Table 2. Effect of fungicides, organic oils and a detergent sprayed against powdery mildew of jujube on number of spotted fruit at two locations during 2007-08 and 2008-09.

\begin{tabular}{|c|c|c|c|c|c|c|c|c|}
\hline \multirow{4}{*}{ Treatment } & \multicolumn{8}{|c|}{ Number of spotted fruit per plant } \\
\hline & \multicolumn{4}{|c|}{ Mawna, Gazipur } & \multicolumn{4}{|c|}{ Ishurdi, Pabna } \\
\hline & \multicolumn{2}{|c|}{ 2007-08 } & \multicolumn{2}{|c|}{ 2008-09 } & \multicolumn{2}{|c|}{ 2007-08 } & \multicolumn{2}{|c|}{ 2008-09 } \\
\hline & Apple Kul & BAU Kul & Apple Kul & BAU Kul & Apple Kul & BAU Kul & Apple Kul & BAU Kul \\
\hline Thiovit (0.3\%) & $21.00 \mathrm{~cd}$ & 6.67 de & $13.33 \mathrm{~cd}$ & $8.33 \mathrm{~d}$ & $19.00 \mathrm{~cd}$ & $8.67 \mathrm{c}$ & $15.33 \mathrm{bc}$ & $9.00 \mathrm{~b}$ \\
\hline Tilt (0.05\%) & $19.00 \mathrm{~d}$ & $4.33 \mathrm{e}$ & $11.67 \mathrm{~d}$ & $8.00 \mathrm{~d}$ & $15.33 \mathrm{~d}$ & $8.33 \mathrm{c}$ & $12.67 \mathrm{c}$ & $8.67 \mathrm{~b}$ \\
\hline Folicur (0.05\%) & $19.00 \mathrm{~d}$ & 5.67 de & $12.00 \mathrm{~d}$ & $9.00 \mathrm{~cd}$ & $18.33 \mathrm{~d}$ & $9.00 \mathrm{c}$ & $13.00 \mathrm{c}$ & $9.33 \mathrm{~b}$ \\
\hline Bavistin (0.1\%) & 26.00 abcd & $10.33 \mathrm{~cd}$ & $18.00 \mathrm{abcd}$ & $10.30 \mathrm{bcd}$ & $27.00 \mathrm{bc}$ & $12.00 \mathrm{abc}$ & $16.33 \mathrm{abc}$ & $12.67 \mathrm{ab}$ \\
\hline Cupravit (0.3\%) & 26.33 abcd & $10.67 \mathrm{~cd}$ & 18.67 abcd & $12.67 \mathrm{abc}$ & $29.00 \mathrm{ab}$ & $14.67 \mathrm{abc}$ & $19.33 \mathrm{abc}$ & $13.00 \mathrm{ab}$ \\
\hline Dithane M-45 (0.2\%) & $22.33 \mathrm{bcd}$ & 8.67 cde & $15.33 \mathrm{bcd}$ & $10.00 \mathrm{bcd}$ & $23.33 \mathrm{~cd}$ & $11.00 \mathrm{bc}$ & $14.00 \mathrm{c}$ & $12.33 \mathrm{ab}$ \\
\hline Mustard oil (0.5\%) & $29.00 \mathrm{abc}$ & 13.33 abc & $20.67 \mathrm{abc}$ & $14.00 \mathrm{ab}$ & $30.33 \mathrm{ab}$ & $15.67 \mathrm{ab}$ & $20.00 \mathrm{abc}$ & $14.00 \mathrm{ab}$ \\
\hline Neem oil (0.5\%) & 26.00 abcd & $12.67 \mathrm{bc}$ & 18.33 abcd & $12.00 \mathrm{abcd}$ & $26.67 \mathrm{abc}$ & $13.33 \mathrm{abc}$ & $19.67 \mathrm{abc}$ & $13.33 \mathrm{ab}$ \\
\hline Trix (0.5\%) & $32.00 \mathrm{a}$ & $14.33 \mathrm{ab}$ & $22.00 \mathrm{ab}$ & $14.00 \mathrm{ab}$ & $31.00 \mathrm{ab}$ & $16.33 \mathrm{ab}$ & $22.00 \mathrm{ab}$ & 15.00 a \\
\hline Control (Plain water) & $30.00 \mathrm{ab}$ & $18.00 \mathrm{a}$ & $23.00 \mathrm{a}$ & $14.67 \mathrm{a}$ & $34.33 \mathrm{a}$ & $18.00 \mathrm{a}$ & $23 . .33 \mathrm{a}$ & $17.00 \mathrm{a}$ \\
\hline CV (\%) & 11.16 & 18.09 & 14.66 & 12.73 & 10.79 & 17.19 & 15.09 & 16.31 \\
\hline
\end{tabular}

Figures within the same column with a common letter do not differ significantly $(\mathrm{P}=0.05)$. 


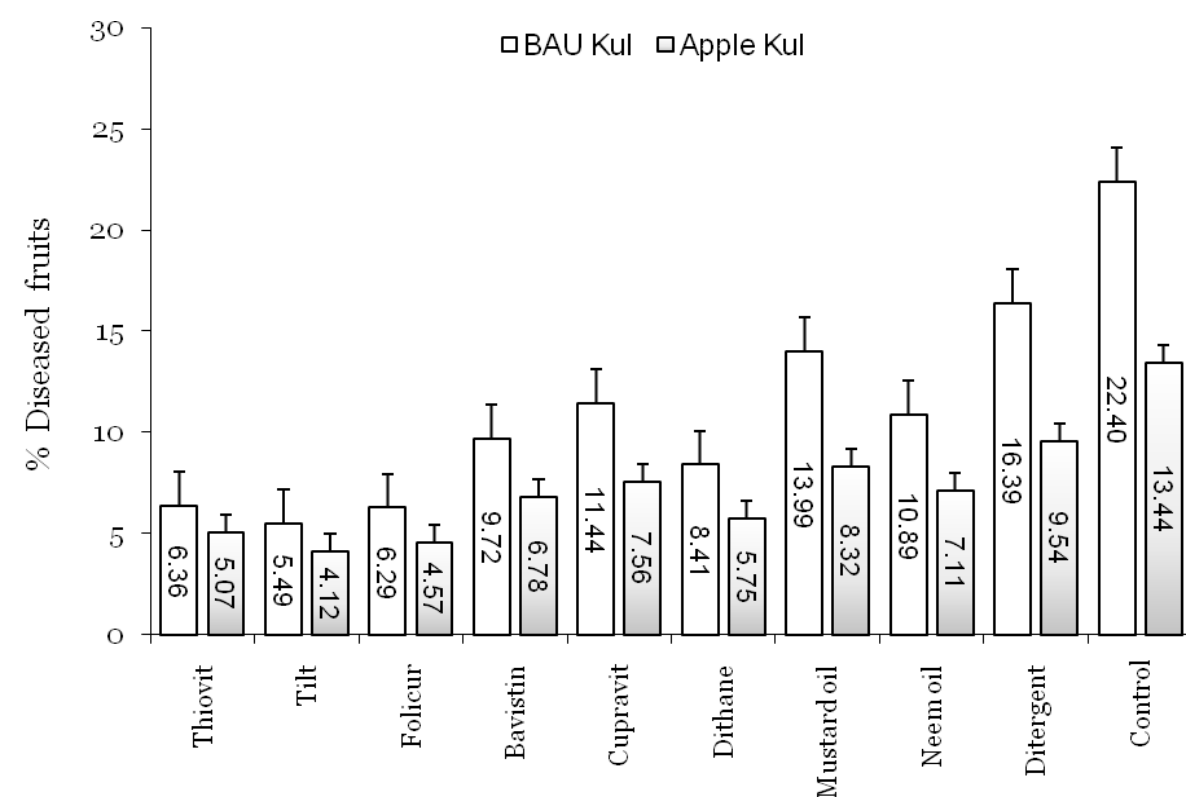

Figure 1. Comparative efficacy of six fungicides, two organic oils and a detergent sprayed against diseases to reduce per cent diseased fruits of jujube.

\section{Total number of fruits per plant}

All treatments significantly increased total fruit number/plant over control except Trix treated plants of Apple Kul at Mawna, Gazipur in 2007-08 and BAU Kul of both locations in cropping year 2008-09. Considering locations and years, Tilt and Thiovit treated plants produced higher total fruit number/plant than other fungicides and oils (Table 3).

\section{Weight of single fruit (g)}

Generally the weight of single fruit was found higher in BAU Kul compared to Apple Kul which may be genetical. Influence of sprays with all fungicides, two organic oils and the detergent on fruit size of variety Apple Kul in two seasons at two locations and that of variety BAU Kul in 2008-09 at Mowna was not significant compared to control. In case of BAU Kul, significant increase in single fruit weight over control was obtained with Tilt and Folicur in 2007-08 at Mowna, in 2008-09 at Ishurdi. In 2007-08 at Ishurdi, significant increase in fruit size of BAU Kul was observed due to spray of only Thiovit and Tilt (Table 4). 
Table 3. Effect of fungicides, organic oils and a detergent sprayed against powdery mildew of jujube on total fruit number per 8 plant at two locations during 2007-08 and 2008-09.

\begin{tabular}{|c|c|c|c|c|c|c|c|c|}
\hline \multirow{4}{*}{ Treatments } & \multicolumn{8}{|c|}{ Total fruit number/plant } \\
\hline & \multicolumn{4}{|c|}{ Mawna, Gazipur } & \multicolumn{4}{|c|}{ Ishurdi, Pabna } \\
\hline & \multicolumn{2}{|c|}{ 2007-08 } & \multicolumn{2}{|c|}{ 2008-09 } & \multicolumn{2}{|c|}{ 2007-08 } & \multicolumn{2}{|c|}{ 2008-09 } \\
\hline & Apple Kul & BAU Kul & Apple Kul & BAU Kul & Apple Kul & BAU Kul & Apple Kul & BAU Kul \\
\hline Thiovit (0.3\%) & $468.30 \mathrm{a}$ & $119.70 \mathrm{ab}$ & $369.00 \mathrm{a}$ & $106.50 \mathrm{ab}$ & 547.70 a & 160.70 a & $417.00 \mathrm{ab}$ & $136.60 \mathrm{ab}$ \\
\hline Tilt (0.05\%) & $440.70 \mathrm{ab}$ & $122.30 \mathrm{a}$ & $374.00 \mathrm{a}$ & $111.00 \mathrm{a}$ & 513.70 a & 162.30 a & $446.00 \mathrm{a}$ & $141.40 \mathrm{a}$ \\
\hline Folicur (0.05\%) & $447.30 \mathrm{ab}$ & $121.00 \mathrm{ab}$ & $357.70 \mathrm{a}$ & $108.90 \mathrm{ab}$ & $535.00 \mathrm{a}$ & $159.30 \mathrm{a}$ & $426.30 \mathrm{ab}$ & $137.90 \mathrm{ab}$ \\
\hline Bavistin (0.1\% & $449.30 \mathrm{ab}$ & $110.00 \mathrm{bc}$ & 354.30 a & $94.97 \mathrm{abc}$ & 518.30 a & $146.30 \mathrm{bc}$ & $390.70 \mathrm{abc}$ & $121.10 \mathrm{bc}$ \\
\hline Cupravit (0.3\%) & $451.30 \mathrm{a}$ & $107.00 \mathrm{~cd}$ & 321.30 a & $92.04 \mathrm{bcd}$ & 525.30 a & $144.30 \mathrm{bc}$ & 370.30 bc & $109.30 \mathrm{~cd}$ \\
\hline Dithane M-45 (0.2\%) & $439.70 \mathrm{ab}$ & $115.70 \mathrm{abc}$ & $374.00 \mathrm{a}$ & $102.20 \mathrm{abc}$ & 524.70 a & $155.00 \mathrm{ab}$ & $387.00 \mathrm{abc}$ & $133.50 \mathrm{~cd}$ \\
\hline Mustard oil (0.5\%) & $449.70 \mathrm{ab}$ & $104.30 \mathrm{~cd}$ & $305.00 \mathrm{a}$ & $86.91 \mathrm{~cd}$ & $500.00 \mathrm{a}$ & $123.30 \mathrm{~d}$ & $368.00 \mathrm{bc}$ & 98.57 de \\
\hline Neem oil (0.5\%) & $443.00 \mathrm{ab}$ & $111.30 \mathrm{abc}$ & 339.70 a & $99.47 \mathrm{abc}$ & 519.00 a & 138.30 c & $386.70 \mathrm{abc}$ & $125.20 \mathrm{abc}$ \\
\hline Trix (0.5\%) & $404.00 \mathrm{ab}$ & $96.33 \mathrm{~d}$ & $302.30 \mathrm{a}$ & 76.28 de & 465.30 a & $116.70 \mathrm{~d}$ & $338.00 \mathrm{c}$ & 83.58 ef \\
\hline Control (Plain water) & $352.70 \mathrm{~b}$ & $79.00 \mathrm{e}$ & $206.30 \mathrm{~b}$ & $61.54 \mathrm{e}$ & $362.30 \mathrm{~b}$ & $91.00 \mathrm{e}$ & $231.70 d$ & $73.16 \mathrm{f}$ \\
\hline CV (\%) & 7.70 & 12.72 & 9.26 & 11.33 & 6.19 & 5.34 & 5.57 & 5.55 \\
\hline
\end{tabular}

Figures within the same column with a common letter do not differ significantly $(\mathrm{P}=0.05)$. 
Table 4. Effect of fungicides, organic oils and a detergent sprayed against powdery mildew of jujube on single fruit weight at two locations during 2007-08 and 2008-09.

\begin{tabular}{|c|c|c|c|c|c|c|c|c|}
\hline \multirow{4}{*}{ Treatment } & \multicolumn{8}{|c|}{ Single fruit wt (g) } \\
\hline & \multicolumn{4}{|c|}{ Mawna, Gazipur } & \multicolumn{4}{|c|}{ Ishurdi, Pabna } \\
\hline & \multicolumn{2}{|c|}{ 2007-08 } & \multicolumn{2}{|c|}{ 2008-09 } & \multicolumn{2}{|c|}{ 2007-08 } & \multicolumn{2}{|c|}{ 2008-09 } \\
\hline & Apple Kul & BAU Kul & Apple Kul & BAU Kul & Apple Kul & BAU Kul & Apple Kul & BAU Kul \\
\hline Thiovit $(0.3 \%)$ & $16.63 \mathrm{a}$ & $92.00 \mathrm{ab}$ & $17.19 \mathrm{a}$ & $91.73 \mathrm{a}$ & $16.42 \mathrm{a}$ & $92.50 \mathrm{ab}$ & $17.67 \mathrm{a}$ & $91.33 \mathrm{abcc}$ \\
\hline Tilt (0.05\%) & $18.00 \mathrm{a}$ & $93.18 \mathrm{a}$ & $17.12 \mathrm{a}$ & $92.83 \mathrm{a}$ & $17.93 \mathrm{a}$ & 93.33 a & $18.17 \mathrm{a}$ & $92.60 \mathrm{a}$ \\
\hline Folicur (0.05\%) & $17.10 \mathrm{a}$ & $92.58 \mathrm{a}$ & $17.29 \mathrm{a}$ & $92.43 \mathrm{a}$ & $17.30 \mathrm{a}$ & $91.50 \mathrm{abc}$ & $17.70 \mathrm{a}$ & $92.20 \mathrm{ab}$ \\
\hline Bavistin (0.1\%) & $16.23 \mathrm{a}$ & 90.33 abc & $17.46 \mathrm{a}$ & $90.33 \mathrm{a}$ & $16.43 \mathrm{a}$ & $89.00 \mathrm{bc}$ & $17.27 \mathrm{a}$ & 90.53 bcd \\
\hline Cupravit (0.3\%) & $16.00 \mathrm{a}$ & 89.83 abc & $17.54 \mathrm{a}$ & $89.87 \mathrm{a}$ & $16.00 \mathrm{a}$ & 88.83 bc & $17.37 \mathrm{a}$ & $90.20 \mathrm{~cd}$ \\
\hline Dithane M-45 (0.2\%) & $16.27 \mathrm{a}$ & 90.58 abc & $17.21 \mathrm{a}$ & $91.57 \mathrm{a}$ & $17.07 \mathrm{a}$ & 90.33 abc & $16.67 \mathrm{a}$ & $91.60 \mathrm{abc}$ \\
\hline Mustard oil (0.5\%) & $16.00 \mathrm{a}$ & 88.83 bc & $16.91 \mathrm{a}$ & $90.00 \mathrm{a}$ & $16.00 \mathrm{a}$ & 89.17 bc & $17.57 \mathrm{a}$ & $89.67 \mathrm{~d}$ \\
\hline Neem oil $(0.5 \%)$ & 16.13 a & 90.43 abc & $16.96 \mathrm{a}$ & $91.83 \mathrm{a}$ & $16.87 \mathrm{a}$ & 90.67 abc & $17.50 \mathrm{a}$ & $91.17 \mathrm{abcc}$ \\
\hline Trix (0.5\%) & $16.00 \mathrm{a}$ & $88.50 \mathrm{abc}$ & $17.34 \mathrm{a}$ & $91.00 \mathrm{a}$ & $16.30 \mathrm{a}$ & 89.50 bc & $17.00 \mathrm{a}$ & $90.50 \mathrm{~cd}$ \\
\hline Control (Plain water) & $16.00 \mathrm{a}$ & $88.03 \mathrm{c}$ & $16.54 \mathrm{a}$ & $90.33 \mathrm{a}$ & $16.00 \mathrm{a}$ & $88.67 c$ & $16.83 \mathrm{a}$ & $90.33 \mathrm{~cd}$ \\
\hline CV (\%) & 4.05 & 1.35 & 6.67 & 1.27 & 6.80 & 1.39 & 3.55 & 0.66 \\
\hline
\end{tabular}

Figures within the same column with a common letter do not differ significantly $(\mathrm{P}=0.05)$. 
Table 5. Effect of fungicides, organic oils and a detergent sprayed against powdery mildew of Jujube on fruit yield per plant at $\mathscr{\infty}$ two locations during 2007-08 and 2008-09.

\begin{tabular}{|c|c|c|c|c|c|c|c|c|}
\hline \multirow{4}{*}{ Treatments } & \multicolumn{8}{|c|}{ Fruit yield (kg/plant) } \\
\hline & \multicolumn{4}{|c|}{ Mawna, Gazipur } & \multicolumn{4}{|c|}{ Ishurdi, Pabna } \\
\hline & \multicolumn{2}{|c|}{ 2007-08 } & \multicolumn{2}{|c|}{ 2008-09 } & \multicolumn{2}{|c|}{ 2007-08 } & \multicolumn{2}{|c|}{ 2008-09 } \\
\hline & Apple Kul & BAU Kul & Apple Kul & BAU Kul & Apple Kul & BAU Kul & Apple Kul & BAU Ku \\
\hline Thiovit (0.3\%) & $7.67 \mathrm{ab}$ & $11.00 \mathrm{abc}$ & $6.50 \mathrm{ab}$ & $9.65 \mathrm{ab}$ & $9.10 \mathrm{a}$ & $14.83 \mathrm{ab}$ & $7.17 \mathrm{abc}$ & $12.70 \mathrm{ab}$ \\
\hline Tilt (0.05\%) & $7.90 \mathrm{a}$ & $11.40 \mathrm{a}$ & $6.77 \mathrm{a}$ & $10.10 \mathrm{a}$ & $9.23 \mathrm{a}$ & $15.13 \mathrm{a}$ & $7.63 \mathrm{a}$ & $13.14 \mathrm{a}$ \\
\hline Folicur (0.05\%) & $7.73 \mathrm{a}$ & $11.17 \mathrm{ab}$ & $6.33 \mathrm{abc}$ & $9.92 \mathrm{ab}$ & $9.13 \mathrm{a}$ & $14.40 \mathrm{abc}$ & $7.37 \mathrm{ab}$ & $12.82 \mathrm{ab}$ \\
\hline Bavistin (0.1\%) & $7.40 \mathrm{ab}$ & 9.83 bcde & $6.03 \mathrm{bcd}$ & $8.66 \mathrm{abc}$ & $8.43 \mathrm{ab}$ & 13.17 bcd & $6.82 \mathrm{abcd}$ & 11.27 bc \\
\hline Cupravit (0.3\%) & $7.20 \mathrm{ab}$ & 9.57 cde & 5.57 bcd & 8.39 bcd & $8.37 \mathrm{ab}$ & $12.80 \mathrm{~cd}$ & $6.50 \mathrm{bcd}$ & 10.17 cd \\
\hline Dithane M-45 (0.2\%) & $7.50 \mathrm{ab}$ & 10.57 abcd & $6.23 \mathrm{abc}$ & $9.32 \mathrm{abc}$ & $8.47 \mathrm{ab}$ & 13.98 abcd & 6.67 abcd & $12.40 \mathrm{ab}$ \\
\hline Mustard oil (0.5\%) & $7.17 \mathrm{ab}$ & 9.27 de & $5.37 \mathrm{~cd}$ & $7.91 \mathrm{~cd}$ & $8.00 \mathrm{~b}$ & 11.00 ef & $6.23 \mathrm{~cd}$ & 9.17 de \\
\hline Neem oil (0.5\%) & $7.47 \mathrm{ab}$ & 10.03 abcd & $5.93 \mathrm{bcd}$ & 9.05 abc & $8.37 \mathrm{ab}$ & 12.57 de & 6.57 abcd & $11.63 \mathrm{abc}$ \\
\hline Trix (0.5\%) & $6.57 \mathrm{bc}$ & 8.37 ef & $5.13 \mathrm{~d}$ & 6.95 de & $7.43 \mathrm{~b}$ & $10.00 \mathrm{f}$ & $5.87 \mathrm{~d}$ & 7.77 ef \\
\hline Control (Plain water) & $5.60 \mathrm{c}$ & $6.93 \mathrm{f}$ & 3.47 e & $5.60 \mathrm{e}$ & $5.77 \mathrm{c}$ & $7.83 \mathrm{~g}$ & 3.83 e & $6.80 \mathrm{f}$ \\
\hline CV (\%) & 13.19 & 14.12 & 10.77 & 13.40 & 11.55 & 4.65 & 12.69 & 9.46 \\
\hline
\end{tabular}

Figures within the same column with a common letter do not differ significantly $(\mathrm{P}=0.05)$. 


\section{Fruit yield (kg/plant)}

Significant increase in fruit yield (kg/plant) was achieved with foliar spray with all fungicides and organic oils tested in the present experiment as compared to control, irrespective of jujube varieties, locations, and seasons. Effectiveness of Thiovit, Tilt, Folicur, and Dithane M-45 to increase fruit yield was statistically similar but comparatively high than other treatments. Trix showed inconsistent results, where significant yield increase was observed at Mowna in 2008-09 in Apple Kul,, at Ishurdi in both seasons in case of Apple Kul, and at Ishurdi in 2007-08 in case of BAU Kul (Table 5). Mean yield increase ranged 33.9068.88\% in Apple Kul and 21.71-83.25\% in BAU Kul. The treatments gave comparatively high yield increase in BAU Kul than in Apple Kul.

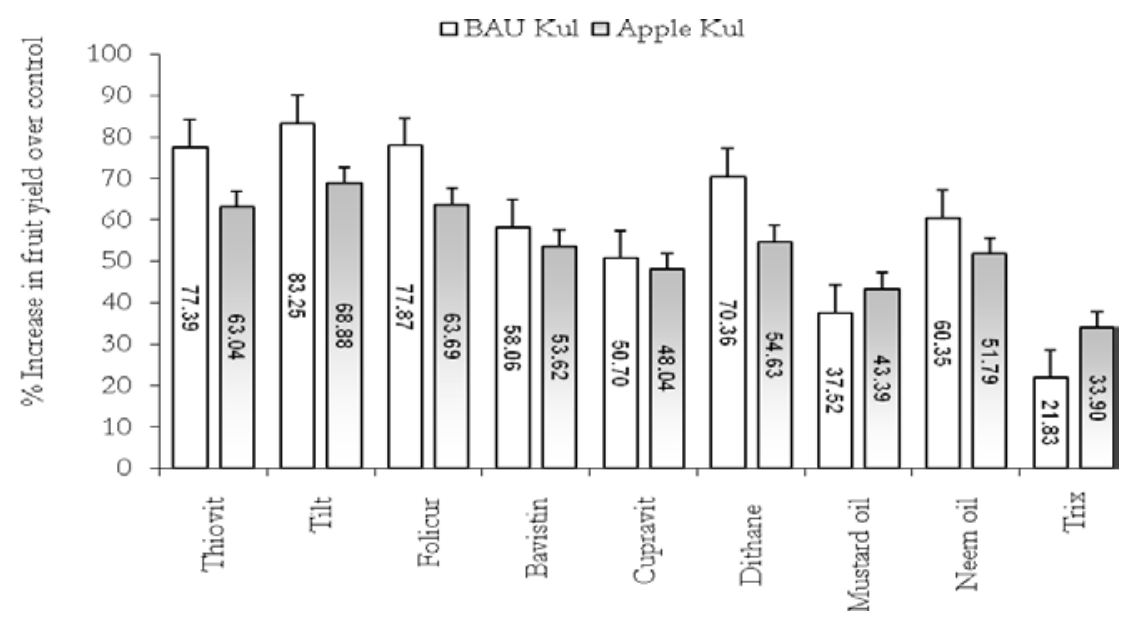

Fig. 2. Comparative efficacy of treatments against powdery mildew disease to increase fruit yield of jujube in average of both years.

\section{Cost and return}

The purpose of marginal analysis is to reveal that how the benefit from investment increases as the amount of investment increase. Economic analysis showed that the highest gross margin (Tk. 377808.13 and Tk 284573.75/ha for BAU Kul and Apple Kul, respectively) was found in Tilt followed by Thiovit, Folicur and Dithane M-45 for both the varieties. On the other hand, MBCR was the highest in Thiovit (23.23 for BAU Kul and 15.61 for Apple Kul) followed by Tilt, Neem oil, and Folicur in both the varieties. It means that Thiovit was the most profitable treatment than others (Table 6 and 7). 
Table 6. Cost and benefit analysis of BAU Kul as influenced by different treatments for management of powdery mildew disease.

\begin{tabular}{l|c|c|c|c|c}
\hline \multicolumn{1}{c}{ Treatment } & $\begin{array}{c}\text { Yield } \\
\text { (kg/ha) }\end{array}$ & $\begin{array}{c}\text { Gross return } \\
\text { (Tk./ha) }\end{array}$ & $\begin{array}{c}\text { Cost of } \\
\text { treatment } \\
\text { (Tk./ha) }\end{array}$ & $\begin{array}{c}\text { Gross margin } \\
\text { (Tk./ha) }\end{array}$ & MBCR \\
\hline Thiovit & 7528.13 & 376406.25 & 7070.00 & 369336.25 & 23.23 \\
Tilt & 7776.56 & 388828.125 & 11020.00 & 377808.13 & 16.03 \\
Folicur & 7548.44 & 377421.875 & 13020.00 & 364401.88 & 12.69 \\
Bavistin & 6707.81 & 335390.625 & 17820.00 & 317570.63 & 6.91 \\
Cupravit & 6395.31 & 319765.625 & 23320.00 & 296445.63 & 4.61 \\
Dithane M-45 & 7229.69 & 361484.375 & 13320.00 & 348164.38 & 11.21 \\
Mustard oil & 5835.94 & 291796.875 & 10520.00 & 281276.88 & 7.57 \\
Neem oil & 6804.69 & 340234.375 & 8520.00 & 331714.38 & 15.03 \\
Trix & 5170.31 & 258515.625 & 8520.00 & 249995.63 & 5.44 \\
Control & 4243.75 & 212187.50 & 0.00 & 212187.50 & - \\
\hline
\end{tabular}

Table 7. Cost and benefit analysis of Apple Kul as influenced by different treatments for management of powdery mildew disease.

\begin{tabular}{l|c|c|c|c|c}
\hline \multicolumn{1}{c|}{ Treatment } & $\begin{array}{c}\text { Yield } \\
\text { (kg/ha) }\end{array}$ & $\begin{array}{c}\text { Gross return } \\
\text { (Tk./ha) }\end{array}$ & $\begin{array}{c}\text { Cost of } \\
\text { treatment } \\
\text { (Tk. /ha) }\end{array}$ & $\begin{array}{c}\text { Gross margin } \\
\text { (Tk./ha) }\end{array}$ & MBCR \\
\hline Thiovit & 4756.25 & 285375.00 & 7070.00 & 278305.00 & 15.61 \\
Tilt & 4926.56 & 295593.75 & 11020.00 & 284573.75 & 10.94 \\
Folicur & 4775.00 & 286500.00 & 13020.00 & 273480.00 & 8.56 \\
Bavistin & 4481.25 & 268875.00 & 17820.00 & 251055.00 & 5.27 \\
Cupravit & 4318.75 & 259125.00 & 23320.00 & 235805.00 & 3.61 \\
Dithane M-45 & 4510.94 & 270656.25 & 13320.00 & 257336.25 & 7.18 \\
Mustard oil & 4182.81 & 250968.75 & 10520.00 & 240448.75 & 7.22 \\
Neem oil & 4428.13 & 265687.50 & 8520.00 & 257167.50 & 10.64 \\
Trix & 3906.25 & 234375.00 & 8520.00 & 225855.00 & 6.97 \\
Control & 2917.19 & 175031.25 & 0.00 & 175031.25 & - \\
\hline
\end{tabular}

Input Price: Thiovit $=$ Tk. 175.00/kg, Tilt $=$ Tk. 1700.00/Litre, Folicur $=$ Tk. 2100.00/Litre, Bavistin = Tk. 1700.00/kg, Diathane M-45 = Tk. 600.00/kg, Cupravit = Tk. 800.00/kg, Mustard oil $=$ Tk. 200.00/Litre, Neem oil $=$ Tk. $150.00 /$ Litre and Trix $=$ Tk. 800.00/Litre

Output price: BAU Kul = Tk 50/kg and Apple Kul = Tk. 60/kg

Findings of the present study revealed that foliar spray with six fungicides and two organic oils effectively decreased severity of powdery mildew and increased fruit yield of jujube irrespective of two varieties in two locations and two years of experimentation. Among the treatments, Tilt, Folicur, Thiovit, and Dithane M-45 were more effective compared to other treatments. They also 
showed effectiveness to reduce number of spotted fruits. Fungicidal spray caused appreciable increase in single fruit weight of the variety BAU Kul, but the treatments did not show any remarkable influence on fruit size of Apple Kul. The most effective fungicide was Tilt followed by Folicur and Thiovit for reducing disease severity and increase fruit yield. Economic analysis also showed that Thiovit, Tilt, Neem oil, and Folicur were the most profitable treatments. Efficacy of fungicides to control powdery mildew of jujube has been reported by many other authors from home and abroad. From Bangladesh, Bakr (2007) reported that Tilt 250 EC (0.05\%), Thiovit (0.2\%), and Bordeaux mixture (1\%) were effective to control powdery mildew disease of jujube. Similar findings have also been reported by other investigators (Bent, 1978; O'Brien et al., 1988; O'Brien, 1994; Singh et al., 1995; Cheah and Page 1997). Yadav et al. (1980) and Singh and Sidhu (1985) found that Carbendazim (0.2\%) can control powdery mildew disease. Sulphur fungicides were found effective for controlling powdery mildew disease by many authors (Kapoor et al., 1975; Gupta et al., 1977, 1978; Yadav and Singh, 1985; Singh et al., 1995). In the present experiment, mustard oil and neem oil were found to be an effective organic pesticide that reduce disease severity as well as increase fruit yield of jujube. Effectiveness of neem oil performed better than mustard oil and it was almost similar to fungicides.

\section{Recommendations}

1) Seven sprays of Tilt 250 EC (Propiconazole) @ 0.05\%, starting from first week of September with an interval of 15 days may be recommended for controlling powdery mildew disease of jujube in commercial orchard.

2) Thiovit (0.3\%) or Folicur 250 EC (Hexaconazole) @ 0.05\% may be applied as an alternate to Tilt $250 \mathrm{EC}$ for controlling powdery mildew disease of jujube in commercial orchard.

3) As organic pesticides, neem oil @ 0.5\% may be applied as a potential alternative of fungicides with same frequency and interval for controlling powdery mildew disease of jujube.

\section{References}

Bakr, M. A. (eds.). 2007. Plant Pathological Research Abstracts. Plant Pathology Division, BARI, Gazipur, 199 Pp.

Bakr, M. A., M. Sakhawat Hossain and H. U. Ahmed (eds). 2010. A Guide to Disease Identification, Data Recording, Rating Scale and Grading System of Major Diseases of Important Crops. 74 Pp.

Bent, K. J. 1978. Chemical control of powdery mildews. In: Spencer DM, ed. The Powdery Mildews. London, UK: Academic Press: 259-280.

Cheah, L. H. and B. B. C. Page. 1997. Screening natural products for the control of powdery mildew in squash. In: Proceedings of the 11th Australasian Plant Pathology Conference. Australia: Australasian Plant Pathology Society, 257. 
Gupta, J. H. Ram Nath and V. P. Srivastava. 1977. Chemical control of powdery mildew of ber (Ziziphus mauritiana Lamk.). Progressive Horticulture 9(1): 81-83.

Gupta, P. C., R. L. Madaan and R. K. Grover. 1978. Occurrence of powdery mildew of ber in Haryana and its control. Indian Phytopath. 31: 440-443.

Homma, Y., Y. Arimoto and T. Misato. 1981. Effect of sodium bicarbonate on each growth stage of cucumber powdery mildew fungus (Sphaerotheca fuliginea) in its life cycle. J. Pesticide Sci. 6(2):201-209.

http://www.weekendgardener.net/plant-diseases/ powdery-mildew-110711.htm

Jamadar, M. M., R. A. Balikai and A. R. Sataraddi. 2009. Status of diseases on ber (Ziziphus mauritiana Lamarck) in India and their management options. Acta Horticulturae (840): 383-390 (http://www.actahort.org/books/840/84053.htm).

Kapoor, S. P., S. S. Cheema and M. P. Singh. 1975. Occurrence and control of powdery mildew of ber (Ziziphus mauritiana Lam.) in Punjab. J. of Res., Punjab Agricultural University 12 (1): 26-29.

Metha, P. R. 1950. Some new diseases of plants of economic importance in Uttar Pradesh. Plant Protection Bulletin, New Delhi, India. 2:50-51

Menzies, J., P. Bowen, D. Ehret, and A. D. M. Glass. 1992. Foliar applications of potassium silicate reduce severity of powdery mildew on cucumber, muskmelon, and zucchini squash. J. American Soc. for Hort. Sci. 117(6):902-905

O'Brien, R. G. 1994. Fungicide resistance in populations of cucurbit powdery mildew (Sphaerotheca fuliginea). New Zealand J. Crop and Hort. Sci. 22(2):145-149; 10

O'Brien, R. G., L. L. Vawdrey and R. J. Glass. 1988. Fungicide resistance in cucurbit powdery mildew (Sphaerotheca fuliginea) and its effect on field control. Australian J. Experimental Agric. 28(3):417-423.

Pareek, O. P. 2001. Fruits for the Future 2: Ber. International Centre for Underutilized Crops, University of Southampton, Southampton, UK.

Reuveni, R. 1995. Suppression of cucumber powdery mildew (Sphaerotheca fuliginea) by foliar sprays of phosphate and potassium salts. Plant Pathology 44(1):31-39

Sharma, S. K., J. K. Dang and R. Datta. 2001. Chemical control of powdery mildew of ber (Zizyphus mauritiana L.). Annals Biol. 17(2): 203-206.

Singh, H. P. and S. S. Sidhu. 1985. Control of ber powdery mildew. Indian Hort. 29(4): 27.

Singh, S. B., S. K. Maheshwar and P. N. Singh. 1995. Field evaluation of fungitoxicants against powdery mildew of ber (Ziziphus mauritiana Lamk.). Annals Plant Protec. Sci. 3(2): 168-169.

Yadav, G. R. and R. Y. Singh. 1985. Effect of different time intervals and fungicides for control of powdery mildew of ber. Indian J. Mycol. and Plant Pathol. 15(3): 261-264.

Yadav, G. R. Nirman, R. S. and Prasad, B. 1980. Powdery mildew of ber in U.P. and its control. Progressive Hort. 12(1): 27-32. 